\title{
Cytochrome b5 reductase 2 is a novel candidate tumor suppressor gene frequently inactivated by promoter hypermethylation in human nasopharyngeal carcinoma
}

\author{
Xue Xiao • Weilin Zhao • Fangyun Tian • Xiaoying Zhou • Jinyan Zhang • Tingting Huang • \\ Bo Hou • Chunping Du • Shumin Wang • Yingxi Mo • Nana Yu • Shiping Zhou • Jinping You • \\ Zhe Zhang • Guangwu Huang • Xianjie Zeng
}

Received: 30 September 2013 / Accepted: 28 November 2013 /Published online: 12 December 2013

(C) The Author(s) 2013. This article is published with open access at Springerlink.com

\begin{abstract}
Cytochrome b5 reductase 2 (CYB5R2), a member of the flavoprotein pyridine nucleotide cytochrome reductase family, is associated with a number of physiological reactions. However, its role in cancer, especially nasopharyngeal carcinoma (NPC), has not been addressed. Here, we investigate the transcript levels and promoter methylation status of CYB5R2 in NPC derived cell lines and tumor biopsies and experimentally address its role as a tumor suppressor gene. We find that CYB5R2 transcript levels are decreased in NPC cell lines and tumor biopsies. Promoter hypermethylation of CYB5R2 was detected in all six tested NPC cell lines and in $84 \%$ of primary NPC tumor biopsies but not in normal nasopharyngeal epithelium. Clinically, CYB5R2 methylation was associated with lymph node metastasis in NPC patients $(P<0.05)$. The endogenous expression of CYB5R2 could be restored in vitro by the methyltransferase inhibitor 5-aza-2'-deoxycytidine in NPC cell lines. Ectopic expression of CYB5R2 had an inhibitory effect on proliferation, clonogenicity and migration of NPC
\end{abstract}

G. Huang and X. Zeng contributed equally to this work.

X. Xiao · W. Zhao · F. Tian • X. Zhou • J. Zhang · T. Huang •

B. Hou $\cdot$ C. Du $\cdot$ S. Wang $\cdot$ N. Yu $\cdot$ S. Zhou $\cdot$ Z. Zhang $\cdot$

G. Huang $(\bowtie)$

Department of Otolaryngology — Head and Neck Surgery, First

Affiliated Hospital of Guangxi Medical University, 6\# Shuangyong

Road, Nanning 530021, China

e-mail: gwhuang@gxmu.edu.cn

Y. Mo

Department of Environmental and Molecular Medicine, Mie

University Graduate School of Medicine, Tsu, Japan

J. You $\cdot X$. Zeng $(\bowtie)$

Department of Head and Neck Surgery, Affiliated Cancer Hospital of

Guangxi Medical University, 71\# Hedi Road, Nanning 530021,

China

e-mail: zengxianjie@gxmu.edu.cn cells. Moreover, in vivo tests in nude mice indicated that ectopic expression of $C Y B 5 R 2$ reduces the tumorigenicity of $C Y B 5 R 2$-negative NPC cells. Collectively, these findings suggest that $C Y B 5 R 2$ may be a functional tumor suppressor gene, frequently inactivated by hypermethylation of its promoter in NPC. We report here the first instance of epigenetic downregulation in NPC tumor biopsies of a key enzyme, CYB5R2, which is responsible for the detoxification of environmental carcinogens. We propose the possibility of utilizing CYB5R2 promoter methylation as a diagnostic biomarker of NPC in the future.

Keywords CYB5R2 - Tumor suppressor gene - Inactivation · Promoter hypermethylation · Nasopharyngeal carcinoma

\section{Introduction}

Nasopharyngeal cancer (NPC) is one of the most common head and neck malignant tumors in Southern China, with an annual incidence rate of $25-50$ per 100,000 person-years, while the frequency in Caucasians in other countries is less than one case per 100,000 person-years $[1,2]$. The etiology of NPC is multifactorial. Accumulated epidemiological and etiological evidence indicate that NPC develops as a result of a complex interaction between genetic factors, exposure to chemical carcinogens, and latent Epstein-Barr virus (EBV) infection [3-6]. Specially, environmental factors such as consumption of salt-preserved fish, cigarette smoking and exposure to environmental chemical pollutants have been reported to be of importance in the development of NPC $[3,7,8]$. EBV is strongly associated with NPC, and it has now been found to function as a tumor promoter rather than an initiator in the tumorigenesis of NPC. But the fact that the majority of the 
human population $(>90 \%)$ carry EBV suggest that host genetic factors might also contribute to the carcinogenesis of NPC, making it develop only in a small subset of the exposed population. However, the relative contribution of these factors in the pathogenesis of NPC remains to be elucidated in detail.

Recent studies demonstrate that genes for carcinogenmetabolizing enzymes may play critical roles in determining individual susceptibility to NPC. Genetic or epigenetic alterations that alter the expression or function of these genes, may decrease the efficiency of the corresponding carcinogen detoxification processes, which, in turn, may increase individual susceptibility and cancer risk. Xenobiotics can be detoxified by phase I or II biotransformation enzymes [9], and genetic polymorphisms in genes encoding biotransformation enzymes such as CYP2E1, GSTM1 and GSTT1 were shown to increase individual susceptibility to NPC [10-13].

Furthermore, growing evidence demonstrates that aberrant epigenetic changes, especially promoter hypermethylation of tumor suppressor genes (TSGs), are important in the multistep carcinogenesis of NPC $[14,15]$. However, no studies have looked into the association of epigenetic alterations in xenobiotic-metabolizing enzymes and NPC.

The human CYB5R2 (cytochrome b5 reductase 2), a 276amino-acid protein, contains one ferredoxin reductase-type flavin adenine dinucleotide-binding domain and belongs to the flavoprotein pyridine nucleotide cytochrome reductase family. It is one of the phase I xenobiotic biotransformation enzymes responsible for detoxification of polycyclic aromatic hydrocarbons and arylhydroxylamine carcinogens, commonly found in cigarette smoke and fried foods [16]. CYB5R2 is involved in various biological processes such as electron transport, oxidation-reduction, lipid metabolism, fatty acid desaturation and/or elongation, cholesterol biosynthesis, drug metabolism, and methemoglobin reduction in erythrocytes [17]. Moreover, it is responsible for reducing both NADHdependent lucigenin chemiluminescence and 2-[4iodophenyl]-3-[4-nitrophenyl]-5-[2, 4-disulfophenyl]-2H-tetrazolium monosodium salt in human spermatozoa [17].

In this study, we evaluated the transcriptional levels and methylation status of CYB5R2 in NPC cell lines and primary tumor biopsies. We further addressed the TSG properties of CYB5R2 in NPC by a series of in vitro and in vivo experiments.

\section{Materials and methods}

NPC cell lines, primary tumor biopsies and normal nasopharyngeal epithelium (NNE)

Six NPC cell lines (CNE1, CNE2, TW03, C666-1, HNE1, and HONE1) were maintained at $37^{\circ} \mathrm{C}$ in the appropriate medium. In total, 70 NPC primary tumor biopsies were collected from newly diagnosed and untreated NPC patients at the department of Otolaryngology - Head and Neck Surgery, First Affiliated Hospital of Guangxi Medical University (Nanning, China), with informed consent from the donors. The diagnosis was established by experienced pathologists according to the World Health Organization (WHO) classification for NPC. Twenty-four NNE tissues obtained by tonsillectomy were included as controls. In all, 50 of the 70 NPC biopsies and 12 of the 24 NNEs were processed for DNA extraction, the rest $20 \mathrm{NPC}$ and $12 \mathrm{NNEs}$ were used for RNA extraction. All biopsy samples were stored in liquid nitrogen. Ethical permission for this study was granted by the Ethical Review Committee of First Affiliated Hospital of Guangxi Medical University, China.

\section{Semi-quantitative RT-PCR}

Preparation of total RNA, first-strand synthesis of cDNA and RT-PCR was performed as described [18]. All primer sequences, annealing temperatures, cycling conditions and expected PCR product sizes are listed in Table 1. Glyceraldehyde-3-phosphate dehydrogenase $(G A P D H)$ was amplified from the same cDNA sample as an internal control. The amplified PCR products were visualized after electrophoresis in $2 \%$ agarose gels and semi-quantitative analysis was performed using Quantity One v4.4.0 software (Bio-Rad, USA).

Methylation-specific PCR (MSP) and bisulfite genomic sequencing (BGS)

The procedure for sodium bisulfite modification of DNA was performed as described $[18,19]$. Bisulfite-modified DNA was amplified using MSP, with primer sets specifically detecting methylated or unmethylated alleles. PCR products were separated on agarose gels. MSP analyses were performed in duplicate. To analyze the methylation status of the targeted region in the CYB5R2 promoter, bisulfite-treated DNA was amplified with a BGS PCR primer set. Amplified PCR products were subcloned and transformed into JM109 competent cells. Several isolated plasmid clones from each cell line or biopsy were sequenced using the BigDye terminator-cycle sequencing kit 3.0 (Applied Biosystems, USA) on an ABI 3100 sequencer.

\section{5-Aza-2'-deoxycytidine (5-aza-dC) treatment}

Four NPC cell lines (CNE1, CNE2, HONE1 and C666-1) were seeded into six-well plates at $2 \times 10^{5}$ cells/well. The next day, cells were treated with the DNA methyltransferase inhibitor 5-aza-dC (Sigma) at $10 \mu \mathrm{M}$ for $96 \mathrm{~h}$, and medium with freshly added 5-aza-dC was replaced every $24 \mathrm{~h}$. Total RNA was extracted for semi-quantitative RT-PCR as described. 
Table 1 Primer sequences, product size and annealing temperature used in this study

\begin{tabular}{|c|c|c|c|}
\hline Primers & Primer sequences & $\begin{array}{l}\text { Product } \\
\text { size (bp) }\end{array}$ & $\begin{array}{l}\text { Annealing } \\
\text { temperature }\end{array}$ \\
\hline \multicolumn{4}{|l|}{ RT-PCR } \\
\hline CYB5R2 - forward & 5'-CCTTGTAGGGACCCGTCCC-3' & 291 & $66^{\circ} \mathrm{C}$ \\
\hline CYB5R2 - reverse & 5'-GACAGGGGTGTAAGCCCTG-3' & & \\
\hline$G A P D H$-forward & 5'-AAGCTCACTGGCATGGCCTT-3' & 375 & $60{ }^{\circ} \mathrm{C}$ \\
\hline$G A P D H$-reverse & 5'-СТCTCTTCCTCTTGTGCTCTTG-3' & & \\
\hline \multicolumn{4}{|c|}{ Methylation-specific PCR } \\
\hline MSP-forward & 5'-GGGGAGCGGGTTAGTCGTC-3' & 140 & $65^{\circ} \mathrm{C}$ \\
\hline MSP-reverse & 5'-GAACCCGCAAACTCGTAACGTC-3' & & \\
\hline USP-forward & 5'-GGGGAGTGGGTTAGTTGTTG-3' & 146 & $58{ }^{\circ} \mathrm{C}$ \\
\hline USP-reverse & 5'-CACCACAAACCCACAAACTC-3' & & \\
\hline \multicolumn{4}{|c|}{ Bisulfite sequencing PCR } \\
\hline BGS-forward & 5'-GGTAGGGTTGATTTAGAGTTAG-3' & 301 & $58{ }^{\circ} \mathrm{C}$ \\
\hline BGS-Reverse & 5'-CTTCAATACTCCATAAATACACC-3' & & \\
\hline
\end{tabular}

Vector construction and transfection

The full-length coding sequence of $C Y B 5 R 2$ from Origene (USA) was subcloned into the pCMV-Tag3A vector (Stratagene, USA). The NPC cell line HONE1, which showed downregulated $C Y B 5 R 2$, was transfected with the pCMV-Tag3A-CYB5R2 plasmid or control pCMV-Tag3A using Lipofectamine 2000 (Invitrogen, USA). Stable clones were obtained by G418 selection $(400 \mu \mathrm{g} / \mathrm{ml})$ for 2 weeks and maintained in $200 \mu \mathrm{g} / \mathrm{ml} \mathrm{G} 418$.

\section{Colony formation assay}

Aliquots of $1 \times 10^{5}$ HONE1 cells were transfected as described above. After $48 \mathrm{~h}$, the cells were transferred to $60-\mathrm{mm}$ cell culture dishes and selected in $400 \mu \mathrm{g} / \mathrm{ml} \mathrm{G} 418$ for 2 weeks. Giemsa-stained colonies were photographed and counted using the Quantity One v4.4.0 software (Bio-Rad, USA). The experiment was performed in triplicate.

\section{Cell proliferation assay}

To determine the cell proliferation rate, stably transfected empty vector-HONE1 and CYB5R2-HONE1 cells were seeded into 96-well plates at $2 \times 10^{3}$ cells per well. Cell density at different time points was measured using the vital stain 3-(4, 5-dimethylthiazol-2-yl)-2,5-diphenyltetrazolium bromide (MTT, Solarbio) and the absorbance (OD490 nm) was measured in a microplate reader (iMark, Bio-Rad, USA). Cells were cultured for 5 days, and five wells from each group were chosen as replicates every $24 \mathrm{~h}$.

Wound healing assay

Empty vector-HONE1 and CYB5R2-HONE1 cells $\left(5 \times 10^{5}\right.$ per well) were seeded in six-well plates and allowed to adhere for
$24 \mathrm{~h}$. Confluent monolayers of cells were scratched by a sterile $1 \mathrm{ml}$ micropipette tip. Photographs were taken at 0 and $24 \mathrm{~h}$ at the same position of the wound and the regrowth of cells into the wound area was measured. The experiment was performed in triplicate.

In vivo tumor growth assay

Eight female 6-week-old Balb/c athymic nude mice (Experimental Animal Center of Guangxi Medical University, China) were used. The experimental protocol for tumor formation in nude mice was approved by the Ethical Review Committee of First Affiliated Hospital of Guangxi Medical University, and the committee's ethical guidelines for animal experimentation were followed. A total of $2 \times 10^{6}$ HONE1 cells, stably transfected with $C Y B 5 R 2$, were subcutaneously injected into the right flank of the nude mice. An equal amount of empty vector-HONE1 cells were injected into the left flank of the mice as a control. The growth of tumors was monitored every 2 days for 2 weeks after inoculation. Tumor volume $(V)$ was calculated as $V=(\pi / 6) L \times W \times H$, where $L, W$, and $H$ represent tumor diameters in three mutually orthogonal planes. The tumor was removed and weighed on day 14.

Statistical analysis

Statistical analysis was performed using SPSS v16 (SPSS Inc., Chicago, IL, USA). Data are shown as mean \pm SD. The association of promoter methylation status with clinical pathological features of NPC patients was analyzed by Pearson chi-square or Fisher's exact test. Paired $t$ test was used to compare in vivo experiment groups. A $P$ value $<0.05$ was considered statistically significant. 


\section{Results}

Inactivation of $C Y B 5 R 2$ in NPC cell lines and primary tumors

To investigate the expression of CYB5R2 in NPC cell lines and NPC biopsies, semi-quantitative RT-PCR was performed. As demonstrated in Fig. 1a, CYB5R2 mRNA expression was undetectable in NPC cell lines CNE1, HONE1, and HNE1 while weak expression was detected in CNE2, TW03, and C666-1. As well, CYB5R2 expression was downregulated in the 20 NPC primary tumor biopsies but easily detected in all NNE samples (Fig. 1b).

The CYB5R2 promoter is hypermethylated in NPC cell lines and primary tumors

Having established that $C Y B 5 R 2$ was downregulated in NPC cell lines and primary biopsies, we further investigated the promoter methylation status of $C Y B 5 R 2$. MSP results showed that the $C Y B 5 R 2$ promoter was hypermethylated in the six NPC cell lines (CNE1, CNE2, TW03, HONE1, HNE1 and C666-1; see Fig. 2a) and in $84 \%$ (42/50) of NPC primary tumors but in none of the 12 NNE samples (Fig. 2b). Unmethylated amplicons were detected in some primary NPC biopsy samples but were most likely due to contaminating non-malignant cells such as stromal cells in a fraction of the tissue samples.

CpG sites in the $C Y B 5 R 2$ promoter are heavily methylated in NPC cell lines and NPC biopsies

To reveal the detailed methylation status of individual $\mathrm{CpG}$ sites, we analyzed the DNA sequence of the promoter region
-389 to -89 bp of $C Y B 5 R 2$ using BGS in two NPC cell lines (TW03 and CNE2), two NPC biopsies (NPC15 and NPC27) and one normal biopsy (NNE7) (Fig. 2c). All 39 CpG sites were heavily methylated in TW03 and CNE2, in agreement with our finding of downregulated CYB5R2 expression in these NPC cell lines. As well, the two NPC biopsies tested showed intense methylation. The presence of some unmethylated $C Y B 5 R 2$ promoter sequences among the clones originating from the two NPC cell lines TW03 and CNE2 may be due to heterogeneity in the original cell lines. The NNE sample was nearly completely free of methylation. The BGS data also demonstrate that our bisulfite conversion of genomic DNA was complete which supports the reliability of our MSP results.

Transcription of CYB5R2 could be restored by 5-aza-dC treatment

The expression of $C Y B 5 R 2$, which was silenced or downregulated in the NPC cell lines CNE1, CNE2, HONE1 and C6661, could be restored after $96 \mathrm{~h} \mathrm{5-aza-dC} \mathrm{treatment} \mathrm{(Fig.} \mathrm{2d).}$ This provides support for our hypothesis that CYB5R2 downregulation in NPC is an epigenetic event and not due to genetic rearrangement or mutation of the gene or its promoter.

\section{Clinico-pathological significance of CYB5R2 promoter} hypermethylation in NPC

Clinical characters of the 50 NPC patients were collected, and we found a significant association of CYB5R2 promoter hypermethylation with the clinico-pathological variable lymph node metastasis $(P<0.05)$, however, there was no
Fig. 1 Expression of cytochrome b5 reductase 2 (CYB5R2) in NPC cell lines, NPC biopsies and NNE samples. a RT-PCR analysis of expression of CYB5R2 in NPC cell lines and NNEs. PC: positive control. GAPDH was the internal control. Water was the blank control. b Semi-quantitative RT-PCR of CYB5R2 expression in NNEs and primary NPC tumors. CYB5R2 expression was normalized to that of GAPDH
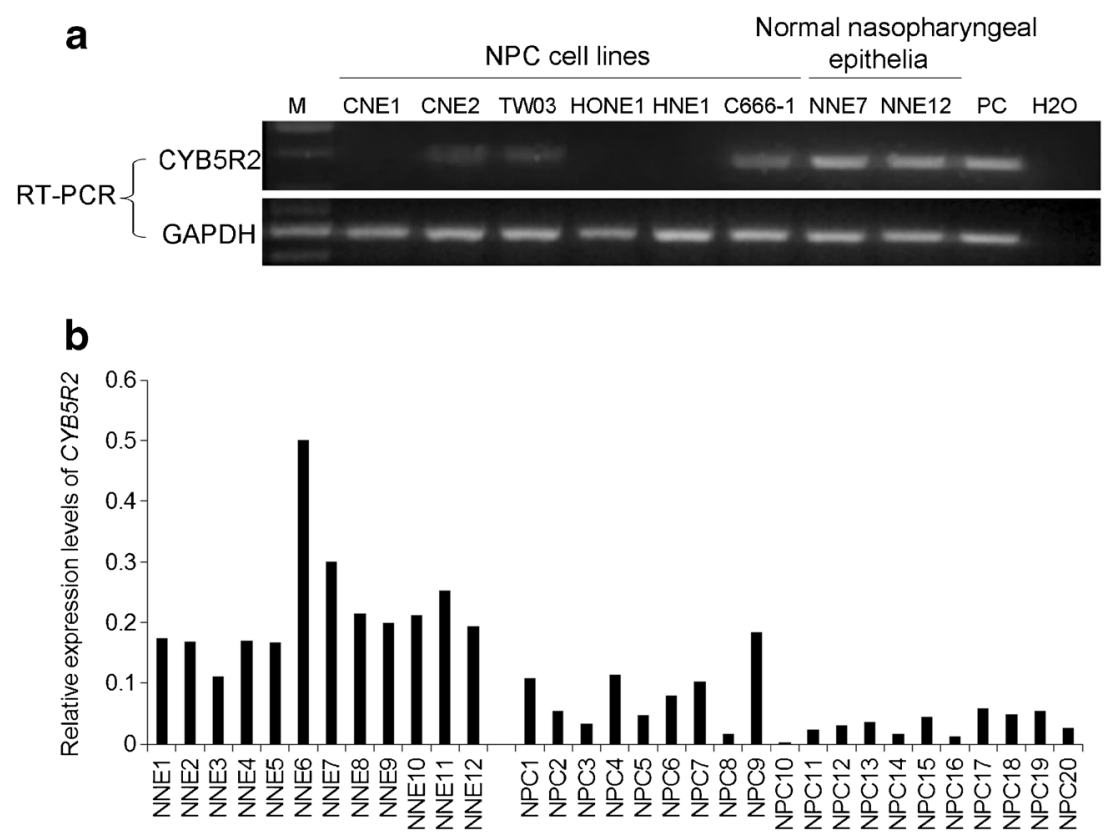
a

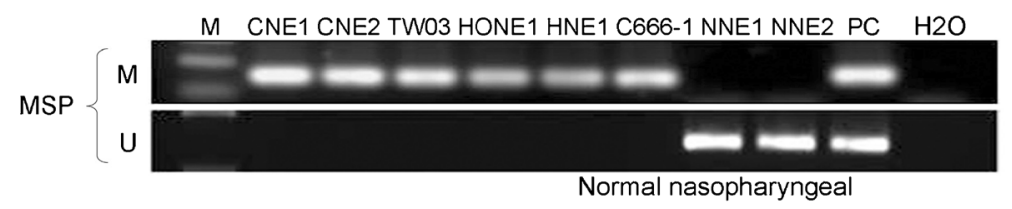

b
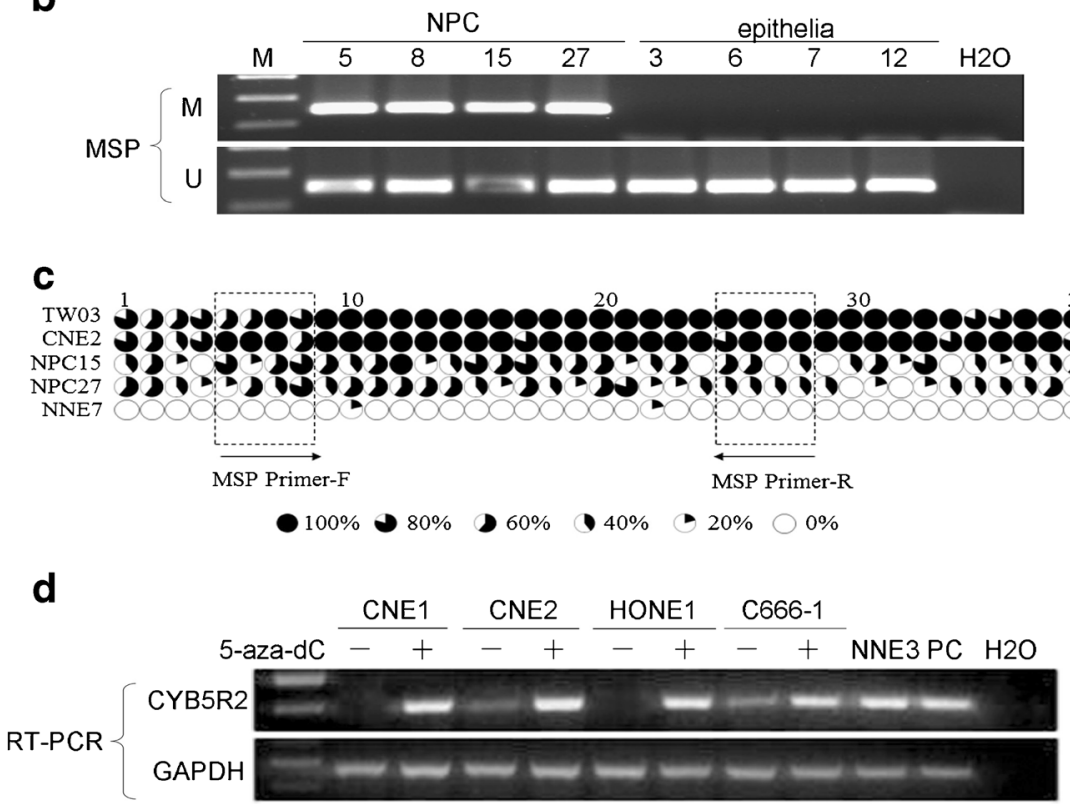

Fig. 2 Analysis of the methylation status of the CYB5R2 promoter region in NPC cell lines, NPC primary tumors and NNE samples. a MSP analysis in NPC cell lines and NNEs. $M$ methylated alleles, $U$ unmethylated alleles. In vitro methylated DNA was used as a methylation-positive control and DNA from normal lymphocytes was the positive control for unmethylated alleles. The blank control was water. b MSP analysis in NPC biopsies and NNE, representative data is shown. c Methylation status of the $39 \mathrm{CpG}$ sites of the CYB5R2 promoter in two
NPC cell lines (TW03 and CNE2), two NPC biopsies (NPC15 and 27) and one NNE biopsy (NNE7). Five clones were randomly selected and sequenced for each sample. Different size filled sectors of the circles represent the fraction of methylated $\mathrm{CpG}$. MSP primer locations are indicated by frames. d Restoration of $C Y B 5 R 2$ expression by treatment with 5-aza-dC in NPC cell lines. One NNE sample and CYB5R2 expression plasmid DNA were used as positive controls significant correlation to age, sex, cancer stage, or pathological subtypes (Table 2).

Reexpression of $C Y B 5 R 2$ in NPC cells suppresses colony formation and cell proliferation and inhibits cell migration

To assess whether CYB5R2 might have properties of a TSG in NPC, we examined the effect of CYB5R2 on clonogenicity and cell proliferation in HONE1 cells. The number of colonies was lower for CYB5R2-HONE1 than empty vector-HONE1 cells $(P<0.05)$ (Fig. 3a). As well, CYB5R2-HONE1 cells grew significantly slower than HONE1 parental and empty vector-HONE1 cells (Fig. 3c). To measure the effect of CYB5R2 reexpression on cell mobility, we used a wound healing assay. Cell movement into the scratched area was slower for CYB5R2-HONE1 than for empty vector-HONE1 cells, which suggests that ectopic expression of CYB5R2 effects a reduction in the motility of NPC cells (Fig. 3b).
Inactivation of CYB5R2 is critical for tumorigenesis in vivo

We further assessed the tumor suppressing capacity of $C Y B 5 R 2$ in vivo. Empty vector-HONE1 and CYB5R2HONE1 cells were injected into the left and right flanks, respectively, of Balb/c athymic nude mice and tumor growth was monitored with 2-day intervals. Mice injected in the flanks with control cells formed larger tumors within 14 days, whereas mice injected in the flanks with $C Y B 5 R 2$-HONE1 showed greatly reduced tumor growth $(P<0.05)$ (Fig. 4). Thus, ectopic expression of $C Y B 5 R 2$ reverses the tumor growth which is associated with its epigenetic downregulation in the HONE1 cells.

\section{Discussion}

NPC has been proposed to be the result of a multistep process involving environmental carcinogens, genetic factors and 
Table 2 Association of CYB5R2 promoter methylation status and clinicopathological characteristics of NPC patients
Data are number of patients

NS not significant

${ }^{\text {a }}$ According to the International Union Against Cancer (UICC)

\begin{tabular}{|c|c|c|c|c|}
\hline & \multirow[t]{2}{*}{ No. of patients } & \multicolumn{2}{|c|}{ Promoter methylation status } & \multirow[t]{2}{*}{$P$ value } \\
\hline & & Methylated & Unmethylated & \\
\hline Age (years) & & & & NS \\
\hline$<60$ & 37 & 30 & 7 & \\
\hline$\geq 60$ & 13 & 10 & 3 & \\
\hline Sex & & & & NS \\
\hline Male & 35 & 28 & 7 & \\
\hline Female & 15 & 11 & 4 & \\
\hline Cancer stage $^{\mathrm{a}}$ & & & & NS \\
\hline I & 4 & 3 & 1 & \\
\hline II & 15 & 12 & 3 & \\
\hline III & 17 & 12 & 5 & \\
\hline IV & 14 & 12 & 2 & \\
\hline Histological subtype & & & & NS \\
\hline Keratinizing squamous cell carcinoma & 7 & 6 & 1 & \\
\hline Non-keratinizing carcinoma & 43 & 35 & 8 & \\
\hline Lymph node metastasis & & & & $P<0.05$ \\
\hline Presence & 33 & 33 & 0 & \\
\hline Absence & 17 & 10 & 7 & \\
\hline
\end{tabular}

latent EBV infection. Among these etiologic factors, EBV is a well-recognized causative factor not only for NPC, but for a number of human malignancies [20]. However, although EBV infects and persists in about $90 \%$ of the human population, NPC develops in only a minority of EBV-infected individuals, which suggests that other risk factors may be involved in disease development. Environmental factors such as lifestyle and occupational exposure to chemical carcinogens and tumor promoters have been suggested as cofactors [3]. Examples of the most relevant environmental exposures are Cantonesestyle salted fish, fermented fish sauce and other preserved foods [3, 8]. These foods contain large amounts of $N$ nitrosodimethylamine (NDMA), $N$-nitrosopyrrolidene (NPYR) and $N$-nitrosopiperidine (NPIP), which may
Fig. 3 Ectopic expression of CYB5R2 inhibits colony formation, migration and proliferation of NPC cells. a Colony formation ability of CYB5R2-transfected and empty vector-transfected HONE1 cells. Colony numbers in the bar graph represent the mean $\pm \mathrm{SD}$ of three independent experiments. $\mathbf{b}$ Migration of CYB5R2-transfected and empty vector-transfected HONE1 cells examined by wound healing assay. c Growth curves of CYB5R2-transfected, empty vector-transfected HONE1 cells and parental cells. Data are mean $\pm \mathrm{SD}(n=5) . * P<0.05$ a
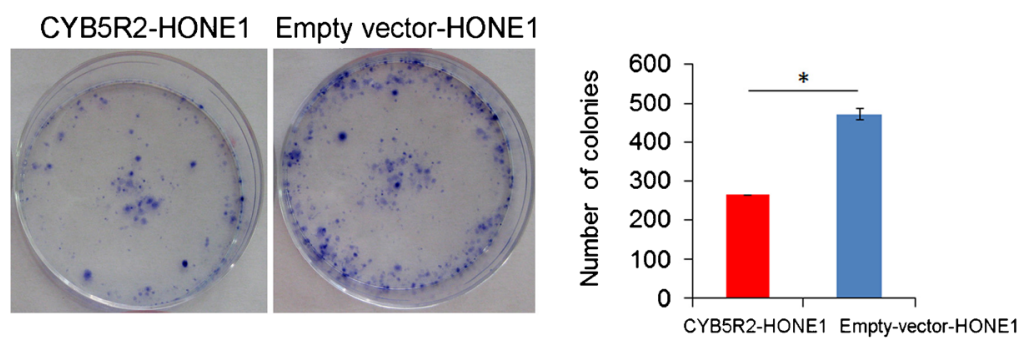

b

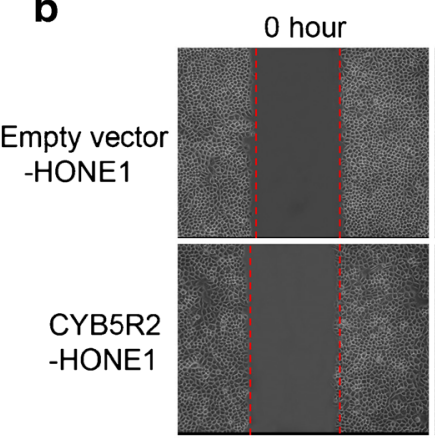

24 hour

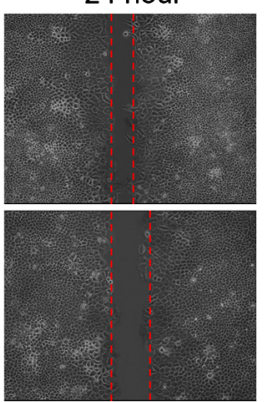

C

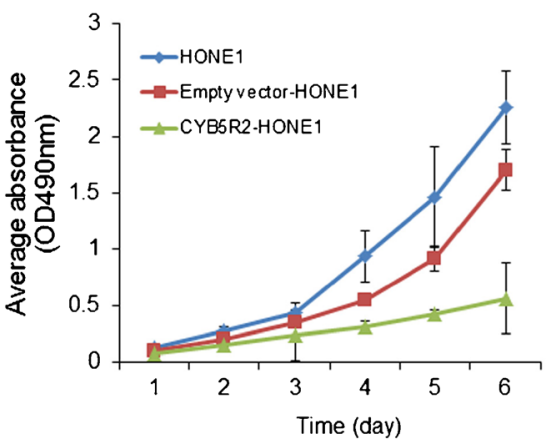


a

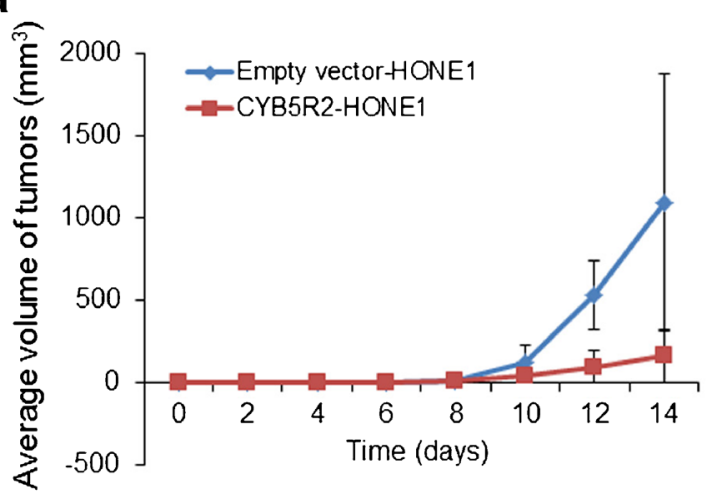

b
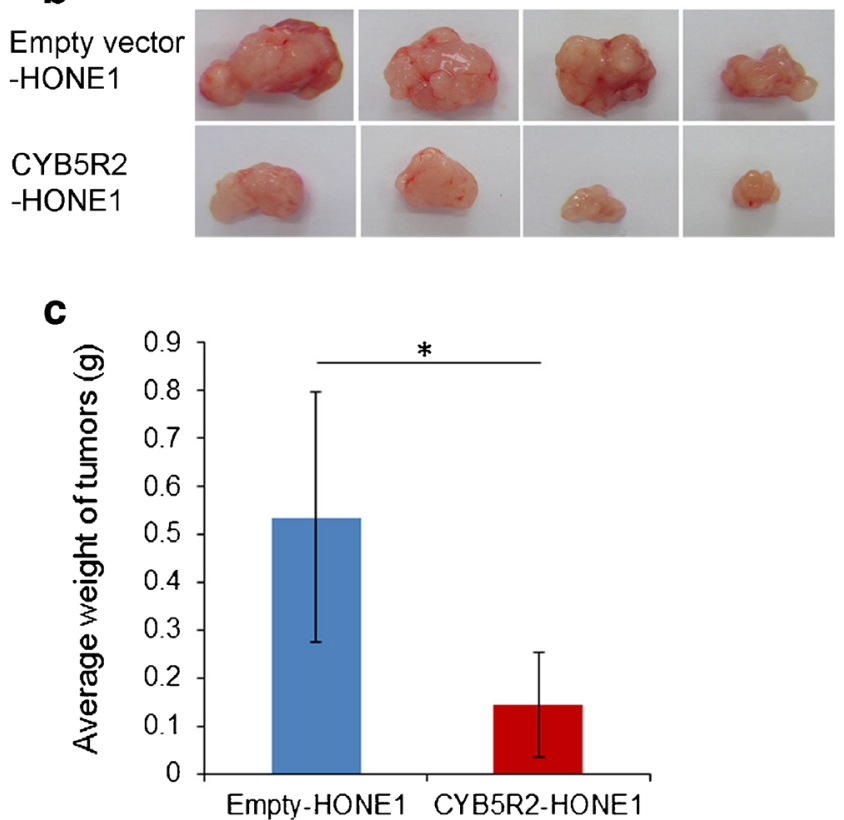

Fig. 4 CYB5R2 reexpression reduces tumorigenicity of NPC cells in nude mice. a Growth curves of tumors in nude mice. The mean volume of tumors from $C Y B 5 R 2$-HONE1 and empty vector-HONE1 cells was evaluated every 2 days after inoculation. Data are mean $\pm \operatorname{SD}(n=8)$. b Representative tumors were removed from the nude mice at day 14 after transplantation. $\mathbf{c}$ Mean $\pm \mathrm{SD}$ weight of tumors $(n=8) .{ }^{*} P<0.05$

contribute to the carcinogenic development in NPC [21]. Occupational exposure to formaldehyde and wood dust are risk factors as well [22-24]. Moreover, cigarette smoke has been consistently found as a moderate risk factor for NPC [7, 25]. Polycyclic aromatic hydrocarbons, nitrosamines and aromatic amines are known carcinogens in cigarette smoke [26, 27]. These aromatic amines can either be activated to carcinogenic aromatic hydroxylamine derivatives in the cytochrome P450 pathway or detoxified by $N$-acetylation in the cytochrome B5 reductase (CYB5R) pathway [16]. The nitrenium or nitroso derivatives produced in the cytochrome P450 activation pathway generate mutagenic DNA adducts while the end products of the CYB5R mediated detoxification pathway are cleared as $\mathrm{N}$-acetylated, water soluble products.
Alterations of genes that encode phase I and phase II xenobiotic biotransformation enzymes [9], involved in metabolic activation or detoxification of potentially carcinogenic substances, have been demonstrated as risk factors for NPC [10-13].

In the present study, we demonstrate that the mRNA expression of a xenobiotic-metabolizing enzyme, CYB5R2, was downregulated in NPC cell lines and primary tumors. The CYB5R2 promoter was aberrantly methylated in all six NPC cell lines $(100 \%)$ and in 42 of $50(84 \%)$ primary NPC tumors, but not in any of the 12 normal controls. Bisulphite genomic sequencing demonstrated that all $39 \mathrm{CpG}$ sites in the tested $C Y B 5 R 2$ promoter were heavily methylated in the NPC cell lines CNE2 and TW03, where CYB5R2 expression is downregulated. A high degree of methylation was also observed in primary NPC tumors. CYB5R2 expression could be restored in four NPC cell lines after treatment with the demethylating agent 5-aza-dC. These results indicate that CYB5R2 was transcriptional inactivation in NPC, and the major mechanism could be a high degree of methylation in its promoter region, which might contribute to the tumorigenesis of NPC.

The expression of CYBSR2 has been evaluated in several human malignancies. Consistent with our findings, microarray analysis revealed that $C Y B 5 R 2$ is downregulated in lobular and ductal invasive breast cancer biopsies and functions as a regulator of cell proliferation, differentiation and transformation [28]. However, these findings are inconsistent with observations in acute B lymphoblastic leukemia, in which CYB5R2 is upregulated [29]. Thus, epigenetic inactivation of CYB5R2 might be tissue-specific events.

Hypermethylated DNA can serve as a biomarker for cancer detection because of its high specificity in distinguishing cancers from normal tissues. We found that the CYB5R2 promoter region was subject to methylation in all of our six NPC cell lines and $84 \%$ of NPC biopsies. Therefore, CYB5R 2 promoter hypermethylation is a frequent event in NPC and not just a phenomenon associated with in vitro cell culture. In addition, epigenetic silencing of $C Y B 5 R 2$ was a tumorspecific event and could be detected in both early- and advanced-stage NPC biopsies, which suggests that $C Y B 5 R 2$ promoter hypermethylation might contribute both to the initiation and progression of NPC. CYB5R2 methylation could serve as a potential biomarker for early diagnosis of NPC. However, due to the lack of patients' follow-up study, we suffered from the limitation that no correlation between the expression of CYB5R2 and the survival rate of NPC patients was verified.

Lymph node metastasis is one of the most significant features of NPC [30]. It is strongly correlated to reduced survival rate and increased risk of tumor recurrence. Identification of biomarkers for lymph node metastasis in NPC would be helpful in designing optimized and individualized therapeutic regimens for NPC. Our finding that CYB5R2 promoter 
methylation is associated with lymph node metastasis in NPC, suggests that $C Y B 5 R 2$ promoter methylation may serve as a diagnostic indicator of lymph node metastasis, which would have great value for the clinical evaluation of NPC cases.

We show that ectopic expression of CYB5R2 significantly inhibited cell proliferation, colony formation and migration of NPC cells in vitro. Moreover, exogenous expression of CYB5R2 inhibits tumorigenicity of NPC cells in vivo in a mouse model system. This evidence strongly implies that CYB5R2 is a putative TSG and plays a role in nasopharyngeal carcinogenesis. To our knowledge, this is the first report of a xenobiotic-metabolizing enzyme functioning as a tumor suppressor in NPC.

In summary, our data demonstrate for the first time that CYB5R2 is epigenetically inactivated by promoter hypermethylation in a human cancer, NPC. Our experimental data provide support for CYB5R2 as a novel and important candidate TSG in NPC. We revealed a frequent and tumor-specific hypermethylation of the CYB5R2 promoter in NPC and a significant association of $C Y B 5 R 2$ promoter hypermethylation with lymph node metastasis, which suggests that the epigenetic state of the CYBSR2 promoter may serve as a diagnostic biomarker and present a possible therapeutic target for NPC.

Acknowledgements We thank Professor Gosta Winberg (Karolinska Institutet, Sweden), for critical comments and suggestions. This study was supported by grants from the National Basic Research Program of China (2011CB504304 and 2012CB526607), Program for New Century Excellent Talents in University (NCET-12-0654), National Natural Scientific Foundation of China (81060167) and Guangxi Natural Science Foundation (2013GXNSFGA019002). The funders had no role in the study design, data collection and analysis, decision to publish, or preparation of the manuscript.

\section{Conflicts of interest None}

Open Access This article is distributed under the terms of the Creative Commons Attribution License which permits any use, distribution, and reproduction in any medium, provided the original author(s) and the source are credited.

\section{References}

1. Tao Q, Chan AT. Nasopharyngeal carcinoma: molecular pathogenesis and therapeutic developments. Expert Rev Mol Med. 2007;9:124 .

2. Jia WH, Huang QH, Liao J, Ye W, Shugart YY, Liu Q, et al. Trends in incidence and mortality of nasopharyngeal carcinoma over a 20 25 year period (1978/1983-2002) in Sihui and Cangwu counties in Southern China. BMC Cancer. 2006;6:178.

3. Chang ET, Adami HO. The enigmatic epidemiology of nasopharyngeal carcinoma. Cancer Epidemiol Biomarkers Prev. 2006;15:176577.

4. Lin TM, Chang HJ, Chen CJ, Cheng YJ, Yang CS, Tu SM, et al. Risk factors for nasopharyngeal carcinoma. Anticancer Res. 1986;6:791-6.
5. Yu MC, Yuan JM. Epidemiology of nasopharyngeal carcinoma. Semin Cancer Biol. 2002;12:421-9.

6. Lo KW, To KF, Huang DP. Focus on nasopharyngeal carcinoma. Cancer Cell. 2004;5:423-8.

7. Xu FH, Xiong D, Xu YF, Cao SM, Xue WQ, Qin HD, et al. An epidemiological and molecular study of the relationship between smoking, risk of nasopharyngeal carcinoma, and Epstein-Barr virus activation. J Natl Cancer Inst. 2012;104:1396-410.

8. Yu MC, Ho JH, Lai SH, Henderson BE. Cantonese-style salted fish as a cause of nasopharyngeal carcinoma: report of a case-control study in Hong Kong. Cancer Res. 1986;46:956-61.

9. Croom E. Metabolism of xenobiotics of human environments. Prog in Mole Biol Transl Sci. 2012;112:31-88.

10. Nazar-Stewart V, Vaughan TL, Burt RD, Chen C, Berwick M, Swanson GM. Glutathione $S$-transferase M1 and susceptibility to nasopharyngeal carcinoma. Cancer Epidemiol Biomarkers Prev. 1999;8:547-51.

11. Tiwawech D, Srivatanakul P, Karalak A, Ishida T. Glutathione $S$ transferase M1 gene polymorphism in Thai nasopharyngeal carcinoma. Asian Pac J Cancer Prev : APJCP. 2005;6:270-5.

12. Jiang Y, Li N, Dong P, Zhang N, Sun Y, Han M, et al. Polymorphisms in GSTM1, GSTTI and GSTP1 and nasopharyngeal cancer in the east of China: a case-control study. Asian Pac J Cancer Prev : APJCP. 2011;12:3097-100.

13. Hildesheim A, Anderson LM, Chen CJ, Cheng YJ, Brinton LA, Daly $\mathrm{AK}$, et al. Cyp2e1 genetic polymorphisms and risk of nasopharyngeal carcinoma in Taiwan. J Natl Cancer Inst. 1997;89:1207-12.

14. Lung HL, Cheung AK, Ko JM, Cheng Y, Stanbridge EJ, Lung ML. Deciphering the molecular genetic basis of npc through functional approaches. Semin Cancer Biol. 2012;22:87-95.

15. Lo KW, Chung GT, To KF. Deciphering the molecular genetic basis of NPS through molecular, cytogenetic, and epigenetic approaches. Semin Cancer Biol. 2012;22:79-86.

16. Kurian JR, Chin NA, Longlais BJ, Hayes KL, Trepanier LA. Reductive detoxification of arylhydroxylamine carcinogens by human NADH cytochrome b5 reductase and cytochrome b5. Chem Res Toxicol. 2006; 19:1366-73.

17. Sofos E, Pescosolido MF, Quintos JB, Abuelo D, Gunn S, Hovanes $\mathrm{K}$ et5 al. A novel familial 11p15.4 microduplication associated with intellectual disability, dysmorphic features, and obesity with involvement of the ZNF214 gene. Am J Med Genet A. 2012;158A:50-8.

18. Zhang Z, Sun D, Van do N, Tang A, Hu L, Huang G. Inactivation of RASSF2A by promoter methylation correlates with lymph node metastasis in nasopharyngeal carcinoma. Int J Cancer. 2007;120: 32-8.

19. Olek A, Oswald J, Walter J. A modified and improved method for bisulphite based cytosine methylation analysis. Nucleic Acids Res. 1996;24:5064-6.

20. Raab-Traub N. Novel mechanisms of EBV-induced oncogenesis. Curr Opin Virol. 2012;2:453-8.

21. Poirier S, Ohshima H, de-The G, Hubert A, Bourgade MC, Bartsch $H$. Volatile nitrosamine levels in common foods from Tunisia, South China and Greenland, high-risk areas for nasopharyngeal carcinoma (NPC). Int J Cancer. 1987;39:293-6.

22. Vaughan TL, Stewart PA, Teschke K, Lynch CF, Swanson GM, Lyon JL, et al. Occupational exposure to formaldehyde and wood dust and nasopharyngeal carcinoma. Occup Environ Med. 2000;57:376-84.

23. Armstrong RW, Imrey PB, Lye MS, Armstrong MJ, Yu MC, Sani S. Nasopharyngeal carcinoma in Malaysian Chinese: occupational exposures to particles, formaldehyde and heat. Int $\mathrm{J}$ Epidemiol. 2000;29:991-8.

24. Yang XR, Diehl S, Pfeiffer R, Chen CJ, Hsu WL, Dosemeci M, et al. Chinese, American Genetic Epidemiology of NPCST: Evaluation of risk factors for nasopharyngeal carcinoma in high-risk nasopharyngeal carcinoma families in taiwan. Cancer Epidemiol Biomarkers Prev. 2005;14:900-5. 
25. Xue WQ, Qin HD, Ruan HL, Shugart YY, Jia WH. Quantitative association of tobacco smoking with the risk of nasopharyngeal carcinoma: a comprehensive meta-analysis of studies conducted between 1979 and 2011. Am J Epidemiol. 2013;178:325-38.

26. Hecht SS. Tobacco smoke carcinogens and lung cancer. J Natl Cancer Inst. 1999;91:1194-210.

27. Hecht SS. Tobacco smoke carcinogens and breast cancer. Environ and Mole Mutagen. 2002;39:119-26.
28. Turashvili G, Bouchal J, Baumforth K, Wei W, Dziechciarkova M, Ehrmann J, et al. Novel markers for differentiation of lobular and ductal invasive breast carcinomas by laser microdissection and microarray analysis. BMC Cancer. 2007;7:55.

29. Lotem J, Sachs L. Epigenetics and the plasticity of differentiation in normal and cancer stem cells. Oncogene. 2006;25:7663-72.

30. Wei WI, Sham JS. Nasopharyngeal carcinoma. Lancet. 2005;365: 2041-54. 